\title{
Comparison of T-matrix method with discrete sources method applied for total internal reflection microscopy
}

\author{
Norbert Riefler $^{\mathrm{a}, *}$, Elena Eremina ${ }^{\mathrm{a}}$, Christopher Hertlein ${ }^{\mathrm{b}}$, Laurent Helden ${ }^{\mathrm{b}}$, \\ Yuri Eremin ${ }^{\mathrm{c}}$, Thomas Wriedt ${ }^{\mathrm{d}}$, Clemens Bechinger ${ }^{\mathrm{b}}$ \\ ${ }^{a}$ Universität Bremen, Badgasteiner Str. 3, 28359 Bremen, Germany \\ ${ }^{\mathrm{b}}$ Universität Stuttgart, 2. Physikalisches Institut, Pfaffenwaldring 57, 70550 Stuttgart, Germany \\ ${ }^{\mathrm{c}}$ Faculty of Applied Mathematics and Computer Science, Moscow State University, Lenin's Hills, 119992 Moscow, Russia \\ ${ }^{\mathrm{d}}$ Stiftung Institut für Werkstofftechnik, Badgasteiner Str. 3, 28359 Bremen, Germany
}

\begin{abstract}
In the paper we applied a variant of the T matrix method, the null field method with discrete sources (NFM DS) and the discrete sources method (DSM) to model light scattering by a particle near a plane surface in an evanescent wave field. Such investigations have a great practical value for total internal reflection microscopy (TIRM). The numerical algorithms of DSM and NFM DS have been modified to model the specific conditions of real measurement experiments carried out in Stuttgart University. Objective response and scattering cross section have been calculated. Numerical results of both methods have been compared and demonstrate good agreement with measurements.
\end{abstract}

Keywords: Null field method with discrete sources (NFM DS); Discrete sources method (DSM); Evanescent wave scattering; Total internal reflection microscopy (TIRM)

\section{Introduction}

Evanescent wave scattering by a single particle nowadays is widely used in different technical, optical and biological applications. Evanescent wave scattering is the base for especially sensitive microscopic techniques, as it allows to detect scattering from very small objects, whose detection in any other way is very complicated or impossible. One of such techniques is total internal reflection microscopy (TIRM). Since its invention in the mid-eighties [1] TIRM appears to be a suitable and very sensitive technique to measure weak interaction forces between colloidal particles and plane surface [2]. Recent reviews of the TIRM technique can be found in [3-5].

In a TIRM-based experimental setup typically a laser beam propagates in the glass prism and hits the prism-water interface with an angle slightly larger than the angle of total internal reflection. As a result an evanescent field is generated at the interface and decays into the water. If a colloidal particle having a larger refractive index than water is in the vicinity of the interface, it will scatter light from the evanescent field.

\footnotetext{
${ }^{*}$ Corresponding author. Tel.: + 494212183583 ; fax: + 494212185378.

E mail address: riefler@iwt.uni bremen.de (N. Riefler).
} 
The scattered light can be detected by a photomultiplier. Due to the strong dependence of the evanescent fields intensity on the distance from the interface, the scattered light contains information about the distance between particle and interface. Thus single particle evanescent light scattering is an efficient tool to monitor the Brownian motion of spherical colloidal particles perpendicular to the interface with a resolution in the $10 \mathrm{~nm}$ range. However, for correct interpretation of measurement results, precise knowledge of the scattered intensity dependence on the particle-surface distance is required. This can be provided in two independent ways by the light scattering models described here.

To model evanescent light scattering by a particle on a surface we applied two different methods, used by our group: discrete sources method (DSM) [6,7] and a variation of the T-matrix method called null-field method with discrete sources (NFM-DS) [8]. Both methods have been previously applied to model evanescent light scattering [9-11]. To satisfy the specific conditions of real TIRM experiments carried out in Stuttgart University [12] we modified the numerical algorithms to take into account all details, like the presence of a polarizer and the numerical aperture of an objective lens.

To verify the correctness of the calculations of an algorithm we use at least two different methods to model new particle geometries. For this purpose, we describe in the following two methods for calculating TIRM scattering responses. First we will discuss the theoretical foundations of DSM and NFM-DS in the following sections. The computer codes of the methods are completely different and independent of each other. Then we present numerical results and comparisons between both methods and measured experimental results together with a short discussion.

\section{Mathematical formalism}

Consider an axial symmetric particle with interior domain $D_{\mathrm{i}}$ and smooth boundary $\partial D$ placed above the plane surface $\Sigma$. The upper half-space corresponding to the ambient medium is denoted by $D_{0}$, while the lower half-space corresponding to the glass prism is denoted by $D_{1}$. Let us introduce a Cartesian coordinate system $O x y z$ by choosing its origin $O$ at the intersection point of the axis of symmetry of the particle and the plane $\Sigma$. The $z$-axis coincides with the axis of symmetry and is directed into domain $D_{0}$ so that the plane $z=0$ corresponds to the $\Sigma$ plane. Now we would like to describe theoretical basis of both methods.

\subsection{Discrete sources method}

We assume that the exciting field $\left\{\mathbf{E}_{1}^{i}, \mathbf{H}_{1}^{i}\right\}$ is a plane wave propagating from $D_{1}$ at angle $\theta_{1}$ with respect to the $z$-axis and transmitting at the interface following Snell's law.

Then the mathematical statement of the scattering problem can be formulated as follows:

$$
\begin{aligned}
& \nabla \times \mathbf{H}_{\zeta}=\mathrm{j} k \varepsilon_{\zeta} \mathbf{E}_{\zeta} ; \quad \nabla \times \mathbf{E}_{\zeta}=-\mathrm{j} k \mu_{\zeta} \mathbf{H}_{\zeta} \quad \text { in } D_{\zeta}, \zeta=0,1, i, \\
& \mathbf{n} \times\left(\mathbf{E}_{i}-\mathbf{E}_{0}\right)=0, \quad \mathbf{n} \times\left(\mathbf{H}_{i}-\mathbf{H}_{0}\right)=0 \quad \text { at } \partial D, \\
& \mathbf{e}_{z} \times\left(\mathbf{E}_{0}-\mathbf{E}_{1}\right)=0, \quad \mathbf{e}_{z} \times\left(\mathbf{H}_{0}-\mathbf{H}_{1}\right)=0 \quad \text { at } \Sigma .
\end{aligned}
$$

Additionally radiation conditions at infinity have to be considered.

Here, $\mathbf{n}$ is the outward unit normal vector to $\partial D, k=\omega / c$ and $\left\{\mathbf{E}_{\zeta}, \mathbf{H}_{\zeta}\right\}$ stands for the total field in the corresponding domain $D_{\zeta}$. Note that the total field in $D_{0}$ is a superposition of the refracted incident field $\left\{\mathbf{E}_{0}^{\mathrm{i}}, \mathbf{H}_{0}^{\mathrm{i}}\right\}$ and the scattered field $\left\{\mathbf{E}_{0}^{\mathrm{s}}, \mathbf{H}_{0}^{\mathrm{s}}\right\}$. If $\operatorname{Im}\left(\varepsilon_{\zeta}, \mu_{\zeta}\right) \leqslant 0$ (the time dependence for the fields is chosen as $\exp \{\mathrm{j} \omega t\})$ and the particle surface is smooth enough $\partial D \subset C^{(1, \alpha)}$, then the above boundary-value problem is uniquely solvable.

We construct an approximate solution to the scattering problem (4) according to the DSM outlines [7]. The amplitudes of discrete sources (DS) are determined from the boundary conditions at the particle surface, which can be rewritten as

$$
\mathbf{n} \times\left(\mathbf{E}_{i}-\mathbf{E}_{0}^{\mathrm{s}}\right)=\mathbf{n} \times \mathbf{E}_{0}^{\mathrm{i}}, \quad \mathbf{n} \times\left(\mathbf{H}_{i}-\mathbf{H}_{0}^{\mathrm{s}}\right)=\mathbf{n} \times \mathbf{H}_{0}^{\mathrm{i}} \quad \text { at } \partial D .
$$


To construct the fields of dipoles and multipoles analytically satisfying the transmission conditions at the plane interface $\Sigma$ we apply the Green tensor for a stratified interface [6]

$$
\stackrel{\leftrightarrow}{G}\left(\mathbf{M}, \mathbf{M}_{0}\right)=\left[\begin{array}{lll}
g^{e, h} & 0 & 0 \\
0 & g^{e, h} & 0 \\
\frac{\partial f}{\partial x_{M}} & \frac{\partial f}{\partial y_{M}} & g^{h, e}
\end{array}\right] .
$$

An approximate solution takes into account an axial symmetry of the particle and the polarization of an external excitation. Let us consider P-polarized excitation. In this case the external excitation is a transmitted plane wave which accepts the form

$$
\begin{aligned}
& \mathbf{E}_{0}^{0}=T^{\mathrm{P}}\left(-\mathbf{e}_{x} \cos \theta_{0}+\mathbf{e}_{z} \sin \theta_{0}\right) \chi_{0}, \quad \mathbf{H}_{0}^{0}=-T^{\mathrm{P}} n_{0} \mathbf{e}_{y} \chi_{0}, \\
& \chi_{0}=\exp \left[-\mathrm{j} k_{0}\left(x \sin \theta_{0}+z \cos \theta_{0}\right)\right] .
\end{aligned}
$$

Here $\eta_{\varsigma}=\sqrt{ } \varepsilon_{\varsigma} \mu_{\varsigma}$ is the associated refractive index of $D_{\varsigma}, \theta_{0}$ is a refraction angle for the wave coming into $D_{0}$. The refraction coefficient $T_{1,0}^{\mathrm{P}}$ can be written as $T_{1,0}^{\mathrm{P}}=2 n_{1} \cos \theta_{1} /\left(n_{1} \cos \theta_{0}+n_{0} \cos \theta_{1}\right)$. Snell's law in this case gives $\sin \theta_{0}=\left(n_{1} / n_{0}\right) \sin \theta_{1}$. Since $\left|n_{1}\right|>\left|n_{0}\right|$ from a certain incident angle $\theta_{1} \geqslant \theta_{1 C}$ on, $\left|\sin \theta_{0}\right|>1$. Then in the upper half-space an evanescent wave appears, which is concentrated along the interface and exponentially decays along the normal to the interface.

In this context, for P-polarized external excitation we should use the following electric and magnetic vector potentials:

$$
\begin{aligned}
\mathbf{A}_{m, n}^{e, 0} & =\left\{g_{m}^{e}\left(\eta, z_{n}\right) \cos (m+1) \phi ;-g_{m}^{e}\left(\eta, z_{n}\right) \sin (m+1) \phi ;-f_{m+1}\left(\eta, z_{n}\right) \cos (m+1) \phi\right\}, \\
\mathbf{A}_{m, n}^{h, 0} & =\left\{g_{m}^{h}\left(\eta, z_{n}\right) \sin (m+1) \phi ;-g_{m}^{h}\left(\eta, z_{n}\right) \cos (m+1) \phi ;-f_{m+1}\left(\eta, z_{n}\right) \sin (m+1) \phi\right\}, \\
\mathbf{A}_{m, n}^{e, h, 0} & =\left\{0 ; 0 ; g_{m}^{h, e}\left(\eta, z_{n}\right)\right\},
\end{aligned}
$$

where $g_{m}^{e, h}, f_{m}$ Fourier harmonics of the corresponding Green tensor components are given by

$$
\begin{aligned}
& g_{m}^{e, h}\left(\eta, z_{n}\right)=\frac{k_{0}}{\mathrm{j}} Y_{m}^{0}\left(\eta, z_{n}\right)+\int_{0}^{\infty} J_{m}(\lambda \rho) v_{11}^{e, h}\left(z, z_{n}, \lambda\right) \lambda^{1+m} \mathrm{~d} \lambda, \\
& f_{m}\left(\eta, z_{n}\right)=\int_{0}^{\infty} J_{m}(\lambda \rho) v_{31}\left(z, z_{n}, \lambda\right) \lambda^{1+m} \mathrm{~d} \lambda .
\end{aligned}
$$

Here $Y_{m}^{0}\left(\eta, z_{n}\right)=h_{m}^{(2)}\left(k_{0}, R_{\eta z_{0}}\right)\left(\rho / R_{\eta z_{0}}\right)^{m}, h_{m}^{(2)}(\cdot)$ is the cylindrical Hankel function, $(\rho, \varphi, z)$ are the cylindrical coordinates, $\eta=(\rho, z), R_{\eta z_{0}}=\rho^{2}+\left(z-z_{n}\right)^{2}$ while $\left\{z_{n}\right\}_{n=1}^{\infty}$ is a dense set of the source points distributed over a segment $\Gamma_{z}^{0} \in D_{i}$ of the axis of symmetry and the corresponding spectral functions have a form

$$
\begin{aligned}
& v_{11}^{e}\left(z, z_{n}, \lambda\right)=\frac{\mu_{1} K_{0}-\mu_{0} K_{1}}{\mu_{1} K_{0}+\mu_{0} K_{1}} \frac{1}{K_{0}} \exp \left\{-K_{0}\left(z+z_{n}\right)\right\}, \\
& v_{11}^{h}\left(z, z_{n}, \lambda\right)=\frac{\varepsilon_{1} K_{0}-\varepsilon_{0} K_{1}}{\varepsilon_{1} K_{0}+\varepsilon_{0} K_{1}} \frac{1}{K_{0}} \exp \left\{-K_{0}\left(z+z_{n}\right)\right\}, \quad z \geqslant 0, z_{n}>0, \\
& v_{31}\left(z, z_{n}, \lambda\right)=\frac{\left(\varepsilon_{1} \mu_{1}-\varepsilon_{0} \mu_{0}\right)}{\left(\mu_{1} K_{0}+\mu_{0} K_{1}\right)\left(\varepsilon_{1} K_{0}+\varepsilon_{0} K_{1}\right)} \frac{1}{K_{0}} \exp \left\{-K_{0}\left(z+z_{n}\right)\right\},
\end{aligned}
$$

where $K_{\zeta}^{2}=\lambda^{2}-k_{\zeta}^{2}, k_{\zeta}^{2}=k^{2} \varepsilon_{\zeta} \mu_{\zeta}, \zeta=0,1$.

For the total field inside the particle we define the following vector potentials:

$$
\begin{aligned}
\mathbf{A}_{m, n}^{e, i} & =\left\{J_{m}^{i}\left(\eta, z_{n}\right) \cos (m+1) \phi ; J_{m}^{i}\left(\eta, z_{n}\right) s(m+1) \phi ; 0\right\} \\
\mathbf{A}_{m, n}^{h, i} & =\left\{J_{m}^{i}\left(\eta, z_{n}\right) \sin (m+1) \phi ; J_{m}^{i}\left(\eta, z_{n}\right) \cos (m+1) \phi ; 0\right\} \\
\mathbf{A}_{0, n}^{e, h, i} & =\left\{0 ; 0 ; J_{0}^{i}\left(\eta, z_{n}\right)\right\}
\end{aligned}
$$


Here $J_{m}^{i}\left(\eta, z_{n}\right)=j_{m}\left(k_{i}, R_{\eta z_{0}}\right)\left(\rho / R_{\eta z_{0}}\right)^{m}, j_{m}(\cdot)$ is the cylindrical Hankel function, $\left\{z_{n}\right\}_{n=1}^{\infty}$ is a dense set of the points distributed over a segment $\Gamma_{z}^{i} \in D_{i}$ of the axis of symmetry.

Using the following notations:

$$
\mathbf{D}_{1}^{\varsigma}=\left(\frac{j}{k \varepsilon_{\zeta} \mu_{\zeta}} \nabla \times \nabla \times,-\frac{1}{\mu_{\varsigma}} \nabla \times\right)^{\mathrm{T}}, \quad \mathbf{D}_{2}^{\varsigma}=\left(\frac{1}{\varepsilon_{\zeta}} \nabla \times, \frac{j}{k \varepsilon_{\zeta} \mu_{\zeta}} \nabla \times \nabla \times\right)^{\mathrm{T}},
$$

we can represent the approximate solution to the scattering problem for P-polarized excitation as

$$
\left(\begin{array}{c}
\mathbf{E}_{\zeta}^{n} \\
\mathbf{H}_{\varsigma}^{n}
\end{array}\right)=\sum_{m=0}^{M} \sum_{n=1}^{N_{\varsigma}^{m}}\left\{p_{m n}^{\varsigma} \mathbf{D}_{1}^{\varsigma} \mathbf{A}_{m, n}^{e, \zeta}+q_{m n}^{\varsigma} \mathbf{D}_{2}^{\varsigma} \mathbf{A}_{m, n}^{h, \zeta}\right\}+\sum_{n=1}^{N_{\varsigma}^{0}} r_{n}^{\varsigma} \mathbf{D}_{1}^{\varsigma} \mathbf{A}_{0, n}^{e, \zeta} .
$$

The case of an S-polarized excitation can be considered in a similar way [9].

Now we would like to briefly describe the numerical realization of the computational algorithm. As mentioned, the above representation (10) satisfies all the conditions of the scattering problem (1) except the transmission conditions at the particle surface (2). These conditions are used to determine the unknown amplitudes of DS $\left\{p_{m n}^{0, i}, q_{m n}^{0, i}, r_{n}^{0, i}\right\}$. Since the scattering problem geometry is axially symmetric with respect to the $z$-axis and DS are distributed over the axis of symmetry, fulfilling the transmission conditions (2) at surface $\partial D$ can be reduced to a sequential solution of the transmission problems for the Fourier harmonics of the fields. So, instead of matching the fields on the scattering surface, we can match their Fourier harmonics separately thus reducing the approximation problem on the surface to a set of problems enforced at the particle surface generatrix $\mathfrak{I}$. By solving these problems one can determine the DS amplitudes $\left\{p_{m n}^{0, i}, q_{m n}^{0, i}, r_{n}^{0, i}\right\}$.

For the determination of amplitudes the generalized point-matching technique is used [7]. The DSM is a direct method and hence it allows to solve the scattering problem for the entire set of incident angles $\theta_{1}$ and for both polarizations ( $\mathrm{P}$ and $\mathrm{S}$ ) at the same time. Besides, the numerical scheme provides an opportunity to control the convergence of the approximate solution to the exact one by a posterior error estimation [7].

After the amplitudes of the DS are determined, the far field pattern $\mathbf{E}_{\infty}(\theta, \varphi)$ of the scattered field can be calculated. It is determined at the upper part of the unit semi-sphere $\Omega=\left\{0^{\circ} \leqslant \theta<90^{\circ}, 0^{\circ} \leqslant \phi \leqslant 360^{\circ}\right\}$ and is given by

$$
\mathbf{E}_{0}^{\mathrm{s}}(\mathbf{r}) / \mathbf{E}^{0}(0)=\frac{\exp \left\{\mathrm{j} k_{0} r\right\}}{r} \mathbf{F}(\theta, \varphi)+O\left(r^{2}\right), \quad r \rightarrow \infty .
$$

Here $\mathbf{F}^{\mathrm{P}, \mathrm{S}}(\theta, \varphi)=F_{\theta}^{\mathrm{P}, \mathrm{S}} \mathbf{e}_{\theta}+F_{\varphi}^{\mathrm{P}, \mathrm{S}} \mathbf{e}_{\varphi}$;

$$
\begin{aligned}
& \mathbf{e}_{\theta}=\mathbf{e}_{x} \cos \theta \sin \varphi+\mathbf{e}_{y} \cos \theta \sin \varphi-\mathbf{e}_{z} \sin \theta \\
& \mathbf{e}_{\varphi}=-\mathbf{e}_{x} \sin \varphi+\mathbf{e}_{y} \cos \varphi
\end{aligned}
$$

By asymptotical estimation of the Weyl-Sommerfeld integrals involved in (7) the representation of the elements of the far field pattern takes the form of a finite linear combination of elementary functions [9]

$$
\begin{aligned}
& F_{\theta}^{\mathrm{P}}(\theta, \varphi)= \mathrm{j} k_{0} \sum_{m=0}^{M} \cos (m+1) \varphi\left(\mathrm{j} k_{0} \sin \theta\right)^{m} \sum_{n=1}^{N_{0}^{m}}\left\{p_{n, m}^{0} \cos \theta\left[\gamma_{n}^{\prime}+\left(v^{e}-v \sin ^{2} \theta\right) \gamma_{n}\right]\right. \\
&\left.+q_{n, m}^{0}\left(\gamma^{\prime h} \gamma_{n}\right)\right\}-\mathrm{j} k_{0} / \varepsilon_{0} \sin \theta \sum_{n=1}^{N_{0}^{0}} r_{n}^{0}\left(\gamma^{\prime h} \gamma_{n}\right), \\
& F_{\varphi}^{\mathrm{P}}(\theta, \varphi)=-\mathrm{j} k_{0} / \varepsilon_{0} \sum_{m=0}^{M} s(m+1) \varphi\left(\mathrm{j} k_{0} / \varepsilon_{0} \sin \theta\right)^{m} \sum_{n=1}^{N_{0}^{m}}\left\{p_{n, m}^{0} \cos \theta\left(\gamma^{\prime e} \gamma_{n}\right)+q_{n, m}^{0}\left[\gamma_{n}^{\prime}+\left(v^{h}-v \sin ^{2} \theta\right) \gamma_{n}\right] \theta\right\}, \\
& F_{\theta}^{\mathrm{S}}(\theta, \varphi)=\mathrm{j} k_{0} / \varepsilon_{0} \sum_{m=0}^{M} s(m+1) \varphi\left(\mathrm{j} k_{0} \varepsilon_{0} \sin \theta\right)^{m} \sum_{n=1}^{N_{0}^{m}}\left\{p_{n, m}^{0} \cos \theta\left[\gamma_{n}^{\prime}+\left(v^{e}-v \sin ^{2} \theta\right) \gamma_{n}\right]-q_{n, m}^{0}\left(\gamma^{\prime h} \gamma_{n}\right)\right\},
\end{aligned}
$$




$$
\begin{aligned}
F_{\varphi}^{S}(\theta, \varphi)= & -\mathrm{j} k_{0} / \varepsilon_{0} \sum_{m=0}^{M} c(m+1) \varphi\left(\mathrm{j} k_{0} / \varepsilon_{0} \sin \theta\right)^{m} \sum_{n=1}^{N_{0}^{m}}\left\{p_{n, m}^{0} \cos \theta\left(\gamma^{\prime e} \gamma_{n}\right)\right. \\
& \left.-q_{n, m}^{0}\left[\gamma_{n}^{\prime}+\left(v^{h}-v \sin ^{2} \theta\right) \gamma_{n}\right] \theta\right\}+\mathrm{j} k_{0} / \varepsilon_{0} \sin \theta \sum_{n=1}^{N_{0}^{0}} r_{n}^{0}\left(\gamma^{\prime h} \gamma_{n}\right) .
\end{aligned}
$$

Here the associated functions accept the form

$$
\begin{aligned}
& v^{h}=\frac{\mathrm{j} \cos \theta-\kappa}{\mathrm{j} \cos \theta+\kappa}, \quad v^{h}=\frac{\mathrm{j} \varepsilon_{1} \cos \theta-\varepsilon_{0} \kappa}{\mathrm{j} \varepsilon_{1} \cos \theta+\varepsilon_{0} \kappa}, \quad v=\frac{2\left(\varepsilon_{0}-\varepsilon_{1}\right)}{\mathrm{j} \cos \theta+\kappa\left(\mathrm{j} \varepsilon_{1} \cos \theta+\varepsilon_{0} \kappa\right)}, \\
& \kappa=\sqrt{ } \sin ^{2} \theta-\varepsilon_{1} / \varepsilon_{0}, \quad \gamma_{n}=\exp \left\{-\mathrm{j} k_{0} z_{n} \cos \theta\right\}, \quad \gamma_{n}^{\prime}=\exp \left\{\mathrm{j} k_{0} z_{n} \cos \theta\right\} .
\end{aligned}
$$

This circumstance ensures an economical computer analysis of the scattering characteristics in the wave zone.

\subsection{Null-field method with DS}

For modeling the scattering problem in the framework of the NFM-DS we use the same notations as before but we choose the origin $O$ at the distance $z_{0}$ above the plane interface. The angle of the incident wave with respect to the axis of symmetry is $\beta_{1}$, the angle of the refracted wave is $\beta_{0}$. Since, the basic formulae for Mie scattering of evanescent waves result from analytic continuation of the standard case of plane wave excitation, we represent the external excitation as a series of regular spherical vector wave functions (SVWF) $\mathbf{M}_{m n^{\prime}}^{1}\left(k_{0} \mathbf{r}\right)$ and $\mathbf{N}_{m n^{\prime}}^{1}\left(k_{0} \mathbf{r}\right)$, i.e.

$$
\mathbf{E}_{\text {inc }}^{\mathrm{ref}}(\mathbf{r})=\sum_{n^{\prime}=1}^{\infty} \sum_{m=n^{\prime}}^{n^{\prime}} D_{m n^{\prime}}\left[a_{m n^{\prime}}^{\mathrm{ref}} \mathbf{M}_{m n^{\prime}}^{1}\left(k_{0} \mathbf{r}\right)+b_{m n^{\prime}}^{\mathrm{ref}} \mathbf{N}_{m n^{\prime}}^{1}\left(k_{0} \mathbf{r}\right)\right] .
$$

Here $D_{m n^{\prime}}$ is a normalization constant and is given by

$$
D_{m n^{\prime}}=\frac{2 n^{\prime}+1}{4 n^{\prime}\left(n^{\prime}+1\right)} \cdot \frac{\left(n^{\prime}-|m|\right) !}{\left(n^{\prime}+|m|\right) !} .
$$

The expansion coefficients $a_{m n^{\prime}}^{\text {ref }}$ and $b_{m n^{\prime}}^{\text {ref }}$ are the coefficients of the refracted plane wave traveling in the $\left(0, \beta_{0}\right)$ direction and are given by

$$
\begin{aligned}
& a_{m n^{\prime}}^{\mathrm{ref}}=-4 j^{n^{\prime}}\left[\mathrm{j} m \pi_{n^{\prime}}^{|m|}\left(\beta_{0}\right) t_{\|}\left(\beta_{1}\right) E_{\beta_{1}}+\tau_{n^{\prime}}^{|m|}\left(\beta_{0}\right) t_{\perp}\left(\beta_{1}\right) E_{\alpha_{1}}\right], \\
& b_{m n^{\prime}}^{\mathrm{ref}}=-4 j^{n^{\prime}+1}\left[\tau_{n^{\prime}}^{|m|}\left(\beta_{0}\right) t_{\|}\left(\beta_{1}\right) E_{\beta_{1}}-\mathrm{j} m \pi_{n^{\prime}}^{|m|}\left(\beta_{0}\right) t_{\perp}\left(\beta_{1}\right) E_{\alpha_{1}}\right] .
\end{aligned}
$$

In the above equation $E_{\beta_{1}}$ and $E_{\alpha_{1}}$ are the parallel and perpendicular components of the electric field, respectively, $\pi_{n}^{|m|}(\theta)=P_{n}^{|m|}(\cos \theta) / \sin \theta$ and $\tau_{n}^{|m|}(\theta)=\mathrm{d} P_{n}^{|m|}(\cos \theta) / \mathrm{d} \theta$ where $P_{n}^{|m|}(\cos \theta)$ are the associated Legendre polynomials, and $t_{\|}$and $t_{\perp}$ denotes the Fresnel transmission coefficients for parallel and perpendicular polarizations defined by

$$
\begin{aligned}
& t_{\|}\left(\beta_{1}\right)=\frac{2 n_{\mathrm{rel}} \cos \beta_{1}}{\cos \beta_{1}+n_{\mathrm{rel}} \cos \beta_{1}}, \\
& t_{\perp}\left(\beta_{1}\right)=\frac{2 n_{\mathrm{rel}} \cos \beta_{1}}{n_{\mathrm{rel}} \cos \beta_{1}+\cos \beta_{1}},
\end{aligned}
$$

with the relative refractive index $n_{\text {rel }}=n_{0} / n_{1}$.

For $\beta_{1}>\beta_{c}=\arcsin \left(n_{0} / n_{1}\right)$ it follows from Snell's law that $\beta_{0}>1$; hence $\cos \beta_{0}$ becomes purely imaginary.

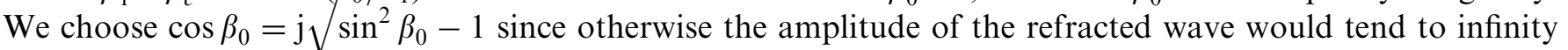
with increasing distance. Note that the expansion (12) is also valid for complex propagation angles if the analytic continuation of the Legendre polynomials to complex values of the argument is considered. The 
scattered field contains the contribution of the direct scattered field

$$
\mathbf{E}_{\mathrm{sca}}^{0}(\mathbf{r})=\sum_{n=1}^{\infty} \sum_{m=n}^{n} D_{m n}\left[e_{m n} \mathbf{M}_{m n}^{3}\left(k_{0} \mathbf{r}\right)+f_{m n} \mathbf{N}_{m n}^{3}\left(k_{0} \mathbf{r}\right)\right]
$$

and the reflected or the interacted field

$$
\mathbf{E}_{\mathrm{sca}}^{R}(\mathbf{r})=\sum_{n=1}^{\infty} \sum_{m=n}^{n} D_{m n}\left[e_{m n} \mathbf{M}_{m n}^{3, R}\left(k_{0} \mathbf{r}\right)+f_{m n} \mathbf{N}_{m n}^{3, R}\left(k_{0} \mathbf{r}\right)\right]
$$

where $\mathbf{M}_{m n}^{3, R}\left(k_{0} \mathbf{r}\right)$ and $\mathbf{N}_{m n}^{3, R}\left(k_{0} \mathbf{r}\right)$ denote the radiating SVWF reflected by the surface. For $\mathbf{r}$ inside a sphere enclosed in the particle and a given azimuthal mode $m$ the reflected SVWF are given by

$$
\left(\begin{array}{c}
\mathbf{M}_{m n}^{3, R}\left(k_{0} \mathbf{r}\right) \\
\mathbf{N}_{m n}^{3, R}\left(k_{0} \mathbf{r}\right)
\end{array}\right)=\sum_{n^{\prime}=1}^{\infty} D_{m n}\left[\left(\begin{array}{c}
\alpha_{m n n^{\prime}} \\
\gamma_{m n n^{\prime}}
\end{array}\right) \mathbf{M}_{m n}^{1}\left(k_{0} \mathbf{r}\right)+\left(\begin{array}{c}
\beta_{m n n^{\prime}} \\
\delta_{m n n^{\prime}}
\end{array}\right) \mathbf{N}_{m n}^{1}\left(k_{0} \mathbf{r}\right)\right] .
$$

Substituting (15) in (16) we get a representation of the interacting field in terms of regular SVWF, i.e.

$$
\mathbf{E}_{\mathrm{sca}}^{R}(\mathbf{r})=\sum_{n^{\prime}=1}^{\infty} \sum_{m=n^{\prime}}^{n} D_{m n^{\prime}}\left[e_{m n^{\prime}}^{R} \mathbf{M}_{m n^{\prime}}^{1}\left(k_{0} \mathbf{r}\right)+f_{m n^{\prime}}^{R} \mathbf{N}_{m n^{\prime}}^{1}\left(k_{0} \mathbf{r}\right)\right]
$$

where

$$
\left(\begin{array}{c}
e_{m n^{\prime}}^{R} \\
f_{m n^{\prime}}^{R}
\end{array}\right)=\sum_{n=1}^{\infty} D_{m n}\left[\left(\begin{array}{c}
\alpha_{m n n^{\prime}} \\
\gamma_{m n n^{\prime}}
\end{array}\right) e_{m n}+\left(\begin{array}{c}
\beta_{m n n^{\prime}} \\
\delta_{m n n^{\prime}}
\end{array}\right) f_{m n}\right] .
$$

In the extended boundary condition method the scattered field coefficients are related to the expansion coefficients of the fields striking the particle by the transition matrix. For an axisymmetric particle the equations become uncoupled, permitting a separate solution for each azimuthal mode. For a fixed azimuthal mode $m$ we truncate the expansions given in (12), (14) and (17) and get the following matrix equation for the scattering problem:

$$
\left[\begin{array}{c}
e_{m n^{\prime}}^{R} \\
f_{m n^{\prime}}^{R}
\end{array}\right]=\left[T_{m n n^{\prime}}\right] \cdot\left(\left[\begin{array}{c}
a_{m n^{\prime}}^{\mathrm{ref}} \\
b_{m n^{\prime}}^{\mathrm{ref}}
\end{array}\right]+\left[\begin{array}{c}
e_{m n^{\prime}}^{R} \\
f_{m n^{\prime}}^{R}
\end{array}\right]\right) .
$$

Here, $m=\overline{-M, M}$ and $n, n^{\prime}=\overline{1, N}$ where $M$ is the number of azimuthal modes and $N$ is the truncation index. The expansion coefficients of the interacting field are related to the scattered field coefficients by a so called reflection matrix $\left[A_{m n^{\prime} n}\right]$, that is,

$$
\left[\begin{array}{c}
e_{m n^{\prime}}^{R} \\
f_{m n^{\prime}}^{R}
\end{array}\right]=\left[A_{m n^{\prime} n}\right] \cdot\left[\begin{array}{c}
e_{m n} \\
f_{m n}
\end{array}\right]
$$

The scattered field coefficients $e_{m n}$ and $f_{m n}$ are obtained by combining matrix equations (18) and (19). The explicit form of the reflection matrix is

$$
\left[A_{m n^{\prime} n}\right]=\left[\begin{array}{ll}
D_{m n} \alpha_{m n n^{\prime}} & D_{m n} \gamma_{m n n^{\prime}} \\
D_{m n} \beta_{m n n^{\prime}} & D_{m n} \delta_{m n n^{\prime}}
\end{array}\right]
$$

The expressions for the elements of the reflection matrix are

$$
\begin{aligned}
& \alpha_{m n n^{\prime}}=4 j^{n^{\prime}} n \int_{0}^{\pi / 2 \mathrm{j} \infty}\left[m^{2} \pi_{n}^{|m|}(\beta) \pi_{n^{\prime}}^{|m|}(\pi-\beta) r_{\|}(\beta)+\tau_{n}^{|m|}(\beta) \tau_{n_{1}}^{|m|}(\pi-\beta) r_{\perp}(\beta)\right] \mathrm{e}^{2 \mathrm{j} k_{0} z_{0} \cos \beta} \sin \beta \mathrm{d} \beta, \\
& \beta_{m n n^{\prime}}=4 j^{n^{\prime}} n \int_{0}^{\pi / 2 \mathrm{j} \infty} m\left[\pi_{n}^{|m|}(\beta) \tau_{n^{\prime}}^{|m|}(\pi-\beta) r_{\|}(\beta)+\tau_{n}^{|m|}(\beta) \pi_{n^{\prime}}^{|m|}(\pi-\beta) r_{\perp}(\beta)\right] \mathrm{e}^{2 \mathrm{j} k_{0} z_{0} \cos \beta} \sin \beta \mathrm{d} \beta,
\end{aligned}
$$




$$
\begin{aligned}
& \gamma_{m n n^{\prime}}=4 j^{n^{\prime}} n \int_{0}^{\pi / 2 \mathrm{j} \infty} m\left[\tau_{n}^{|m|}(\beta) \pi_{n^{\prime}}^{|m|}(\pi-\beta) r_{\|}(\beta)+\pi_{n}^{|m|}(\beta) \tau_{n^{\prime}}^{|m|}(\pi-\beta) r_{\perp}(\beta)\right] \mathrm{e}^{2 \mathrm{j} k_{0} z_{0} \cos \beta} \sin \beta \mathrm{d} \beta, \\
& \delta_{m n n^{\prime}}=4 j^{n^{\prime}} n \int_{0}^{\pi / 2 \mathrm{j} \infty}\left[\tau_{n}^{|m|}(\beta) \tau_{n^{\prime}}^{|m|}(\pi-\beta) r_{\|}(\beta)+m^{2} \pi_{n}^{|m|}(\beta) \pi_{n^{\prime}}^{|m|}(\pi-\beta) r_{\perp}(\beta)\right] \mathrm{e}^{2 \mathrm{j} k_{0} z_{0} \cos \beta} \sin \beta \mathrm{d} \beta,
\end{aligned}
$$

with the Fresnel reflection coefficients

$$
\begin{gathered}
r_{\|}(\beta)=\frac{n_{\mathrm{rel}} \cos \beta-\cos \beta_{0}}{n_{\mathrm{rel}} \cos \beta+\cos \beta_{0}}, \\
r_{\perp}(\beta)=\frac{\cos \beta-n_{\mathrm{rel}} \cos \beta_{0}}{\cos \beta+n_{\mathrm{rel}} \cos \beta_{0}} .
\end{gathered}
$$

The far field scattering patterns are estimated using the amplitude matrix $\mathbf{F}$. The relation to the calculated incident and scattered fields $\mathbf{E}_{\text {sca }}^{R}$ and $\mathbf{E}_{\text {inc }}^{\text {ref }}$ (Eqs. (12) and (17), respectively) are

$$
\left[\begin{array}{c}
E_{\mathrm{sca}, \mathrm{P}}^{R}\left(\theta_{0}, \theta, \varphi\right) \\
E_{\mathrm{sca}, \mathrm{S}}^{\mathrm{ref}}\left(\theta_{0}, \theta, \varphi\right)
\end{array}\right]=\left[\begin{array}{cc}
F_{\theta}^{\mathrm{P}}\left(\theta_{0}, \theta, \varphi\right) & F_{\phi}^{\mathrm{P}}\left(\theta_{0}, \theta, \varphi\right) \\
F_{\theta}^{\mathrm{S}}\left(\theta_{0}, \theta, \varphi\right) & F_{\phi}^{\mathrm{S}}\left(\theta_{0}, \theta, \varphi\right)
\end{array}\right]\left[\begin{array}{c}
E_{\mathrm{inc}, \theta}^{\mathrm{ref}}\left(\theta_{0}, \theta, \varphi\right) \\
E_{\mathrm{inc}, \phi}^{\mathrm{ref}}\left(\theta_{0}, \theta, \varphi\right)
\end{array}\right] .
$$

The main steps of the NFM-DS approach are summarized as follows: calculation of the transition matrix which relates the expansion coefficients of the fields striking the particle to the scattered field coefficients; calculation of the reflection matrix characterizing the reflection of SVWF by the surface; computation of an approximate solution of the governing matrix equation and extrapolation of the scattered field to the far field.

\section{Results and discussion}

Fig. 1 schematically shows the experimental setup typically used for TIRM measurements. A particle within a medium (usually water) is subject to Brownian fluctuations about a distance, e.g. $z$ in Fig. 1, above a prism. It will be illuminated by an evanescent wave, which is caused by the total reflection of the incident wave on the prism-water interface with an angle $\theta_{1}>\theta_{c}, \theta_{c}=\arcsin \left(n_{\text {wat }} / n_{\text {pri }}\right) . \theta_{1}$ in Fig. 1 corresponds to $\beta_{0}$ in our mathematical description of NFM-DS. Recall that $\theta_{1}=\beta_{0}=0^{\circ}$ corresponds to a plane wave propagating normally to the prism surface. More information about the experimental setup and method can be found in [12].

We use an exciting plane wave with a free space wavelength of $\lambda=658 \mathrm{~nm}$ and a glass prism having a refractive index of $n_{\text {pri }}=1.515$. To demonstrate the simulation capabilities of both methods we have chosen polystyrene latex particles with refractive index $n_{i}=1.59$ which are located in water with refractive index

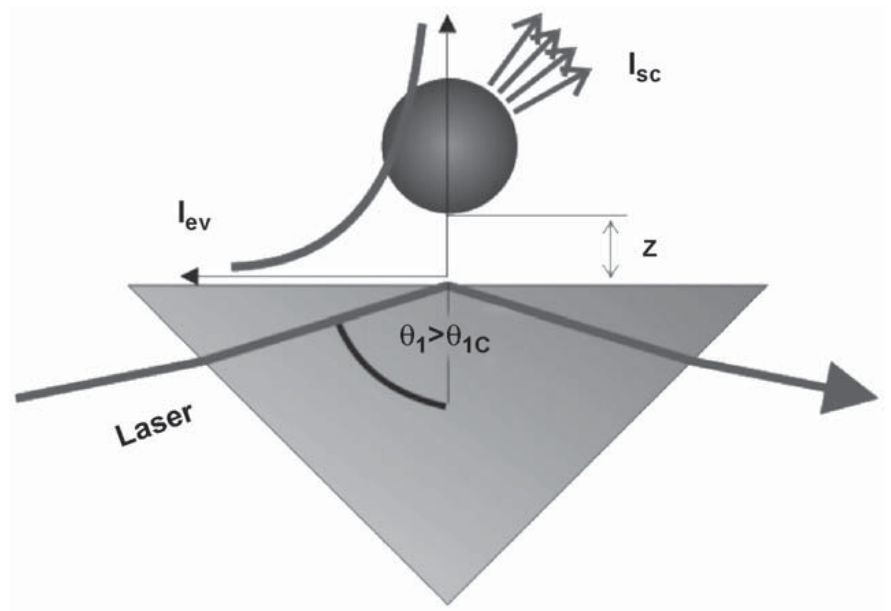

Fig. 1. Measurement setup for TIRM. 
$n_{\text {wat }}=1.333$. So, an evanescent wave appears at incident angles $\theta_{1}>\theta_{c}$. The critical angle corresponding in this particular case is $\theta_{c}=\beta_{c} \approx 61.6264^{\circ}$. The particle-prism distance $z$ is varied from 0 to $1.5 \mu \mathrm{m}$.

One of the most important scattering characteristics is the intensity of scattered light

$$
I^{\mathrm{P}, \mathrm{S}}\left(\theta_{0}, \theta, \varphi\right)=\left|F_{\theta}^{\mathrm{P}, \mathrm{S}}\left(\theta_{0}, \theta, \varphi\right)\right|^{2}+\left|F_{\varphi}^{\mathrm{P}, \mathrm{S}}\left(\theta_{0}, \theta, \varphi\right)\right|^{2},
$$

where $F_{\theta, \varphi}^{\mathrm{P}, \mathrm{S}}\left(\theta_{0}, \theta, \varphi\right)$ are the components of the far field pattern for $\mathrm{P}(11)$ and $\mathrm{S}$ polarized incident waves, in a spherical coordinate system $\theta, \varphi$.

Using a polarizer with a polarizing angle $\varphi$, the desired intensity of the scattered light (26) takes the following forms:

$$
\begin{aligned}
& I_{\mathrm{PP}}=\left|\mathbf{F}_{\mathrm{P}} \cdot \mathbf{e}_{x}\right|^{2}=\left|F_{\theta}^{\mathrm{P}} \cos \theta \cos \varphi-F_{\varphi}^{\mathrm{P}} \sin \varphi\right|^{2} ; \\
& I_{\mathrm{PS}}=\left|\mathbf{F}_{\mathrm{P}} \cdot \mathbf{e}_{y}\right|^{2}=\left|F_{\theta}^{\mathrm{P}} \cos \theta \sin \varphi+F_{\varphi}^{\mathrm{P}} \cos \varphi\right|^{2} ; \\
& I_{\mathrm{SP}}=\left|\mathbf{F}_{\mathrm{S}} \cdot \mathbf{e}_{x}\right|^{2}=\left|F_{\theta}^{\mathrm{S}} \cos \theta \cos \varphi-F_{\varphi}^{\mathrm{S}} \sin \varphi\right|^{2} ; \\
& I_{\mathrm{SS}}=\left|\mathbf{F}_{\mathrm{S}} \cdot \mathbf{e}_{y}\right|^{2}=\left|F_{\theta}^{\mathrm{S}} \cos \theta \sin \varphi+F_{\varphi}^{\mathrm{S}} \cos \varphi\right|^{2} .
\end{aligned}
$$

For example, if $\varphi=0$, the polarizer is parallel to the scattering plane.

In the paper we will also examine the objective response function, which is plotted as a function of particle-prism distance $z$. It represents the detector integrated intensity scattered into the prescribed solid angle of the detector

$$
\sigma_{s}\left(\theta_{0}\right)=\int_{\Omega} I\left(\theta_{0}, \theta, \phi\right) \mathrm{d} \omega
$$

where $\Omega=\left\{0^{\circ} \leqslant \theta<90^{\circ}, 0^{\circ} \leqslant \phi \leqslant 22.08^{\circ}\right.$, which corresponds to the numerical aperture of objective lens $N A=0.5$. The integral response is used to evaluate scattered intensity captured by objective lens [12].

In DSM the number of matching points where the DS amplitudes are defined is increased until the necessary accuracy of the results is achieved. The DS number usually is 2-3 times less than the number of matching points on the particle generatrix. As a rule the DS are deposited on the axis of symmetry inside the particle. The order of multipoles (M) is a priori defined from the condition that the plane wave approximation should be less than $0.1 \%$. The detailed algorithm of matching point's choice and deposition is described in [6].

In NFM-DS the series of SVWF are truncated a priori at an expansion order $n^{\prime}=$ Nrank related to the Wiscombe criterion Nrank $=x+4 x^{1 / 3}+2$ [13], $x$ is the size parameter. The azimuthal index is truncated at $m=0, \pm 1, \pm 2, \ldots, \pm$ Mrank. A convergence procedure runs from $m=0$ to find reliable estimates of Mrank.

In Fig. 2 we compare the scattering diagrams of DSM and NFM-DS for a particle of $d=1.6 \mu \mathrm{m}$ (Mie parameter $x=10$ ). The particle is located at $z=200 \mathrm{~nm}$ above the glass surface. There is perfect agreement between the results from those different programs. Both curves show a maximum of intensity at $\theta=-80^{\circ}$ (SS polarization) and $\theta=-70^{\circ}$ (PP polarization) in the same direction as the incident beam. In the backward direction at about $\theta \geqslant 60^{\circ}$, there are oscillations stemming from interference between light scattering from the particle and the prism-water interface. The small differences of the curves for scattering angels nearly parallel to the interface are not significant because the objective response function for an aperture of $N A=0.5$ $\left(\phi \leqslant 22.08^{\circ}\right)$.

Fig. 3 shows the calculated integrated scattering intensities (Eq. (27)) versus particle-prism distance for a particle with $d=3.0 \mu \mathrm{m}$ (Mie parameter $x=19$ ). One can see a perfect agreement between both methods in this case as well. The intensity maximum of the curves would be expected to be located exactly at the interface $(z=0)$; however, it is located slightly above. Another unexpected result is the oscillations at about $z \approx 0.4 \ldots 0.7 \mu \mathrm{m}$. The oscillations are attributed to scattering interaction between the particle and the plane interface. As a consequence, the intensity does not decay exponentially in this particular case (see the full line in Fig. 3).

In Fig. 4, the calculated scattering intensities (Eq. (27)) versus particle-prism distance are compared to the measured one. The measurements were carried out in Stuttgart University. A detailed description of how the 


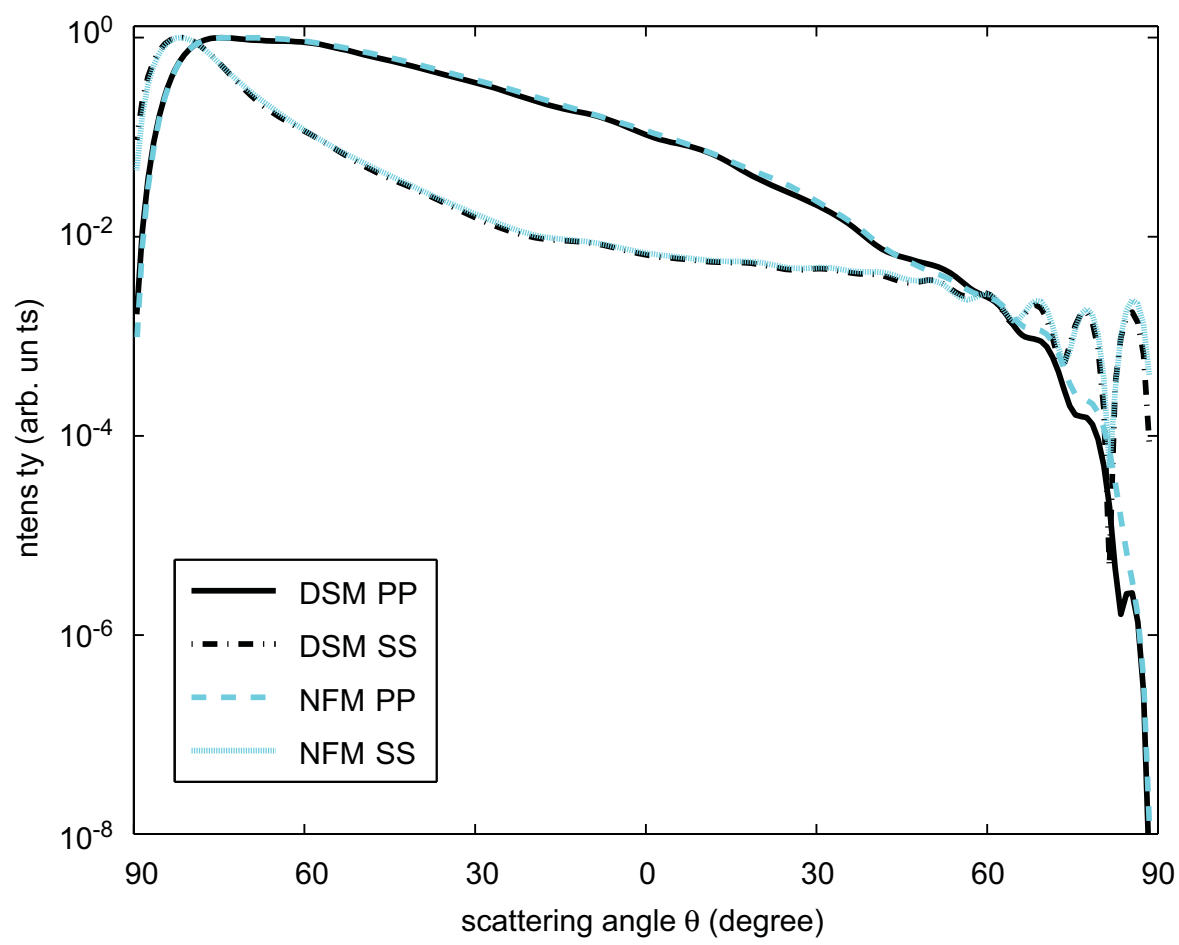

Fig. 2. Scattering diagrams of a latex particle with diameter $d=1.6 \mu \mathrm{m}$; the particle prism distance is $z=200 \mathrm{~nm}$; penetration depth $\zeta=377 \mathrm{~nm}\left(\theta=62.206^{\circ}\right)$.

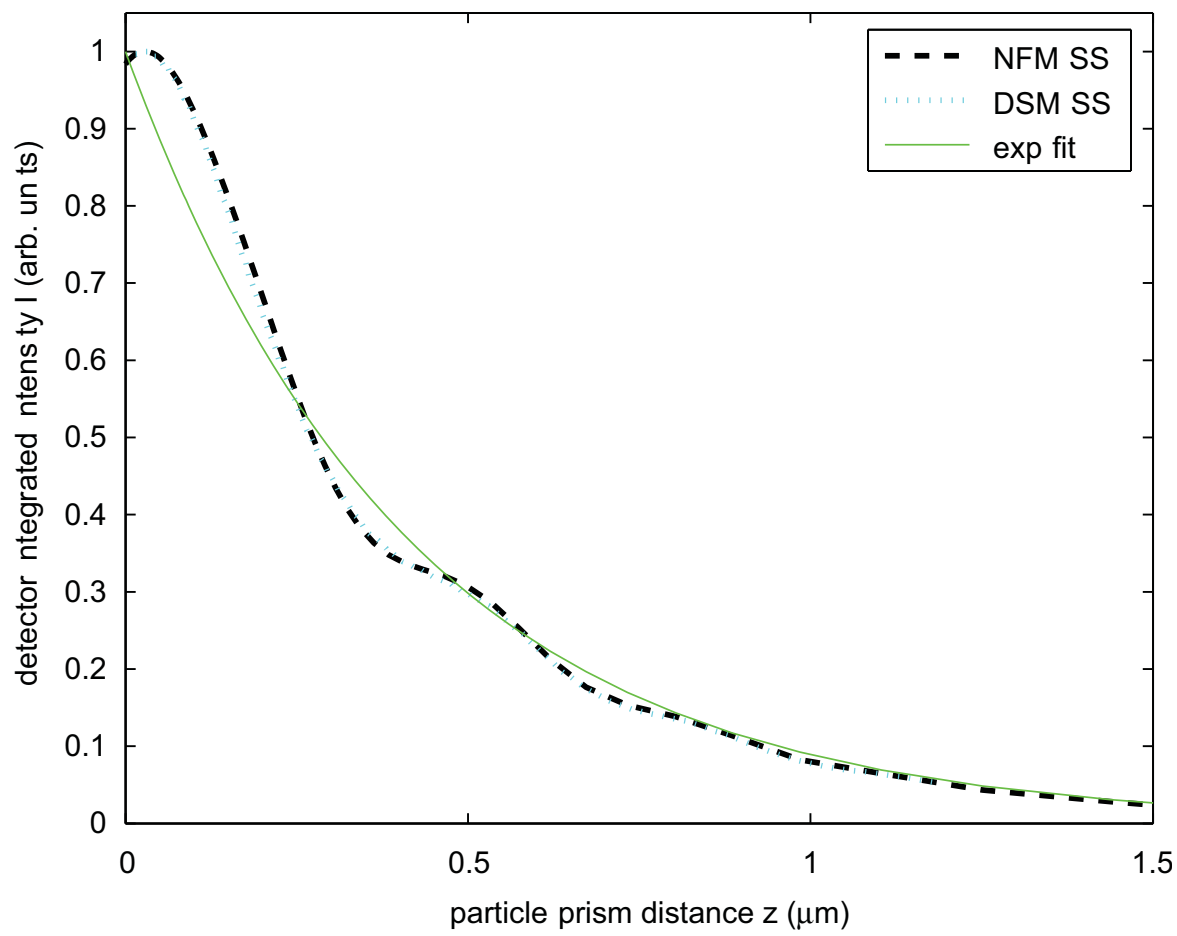

Fig. 3. Detector integrated scattering intensities of a latex particle in water with diameter $d=3.0 \mu \mathrm{m}$; penetration depth $\zeta=400 \mathrm{~nm}$ $\left(\theta=62.141^{\circ}\right)$; the full line is an exponential curve.

intensity distance relation can be obtained from TIRM measurements will be given in a forthcoming article. The used particle diameter in the experiment is $d=1.36 \mu \mathrm{m}$ (Mie parameter $x=8.7$ ). From the comparison one can see that the simulated results are in good agreement with the experimental ones. 


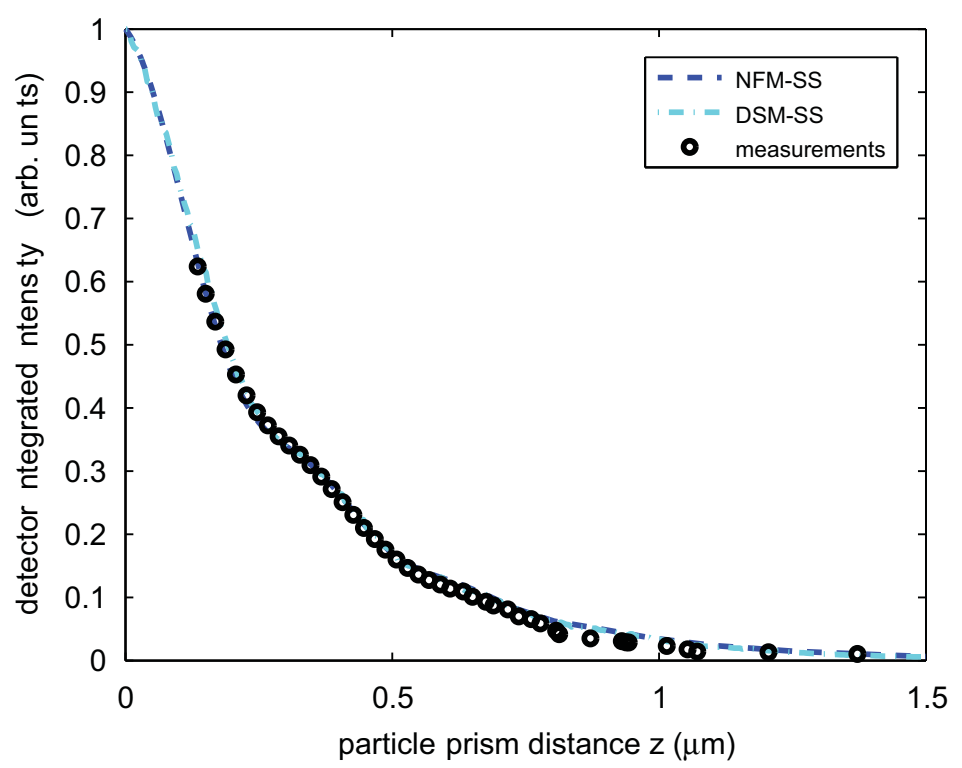

Fig. 4. Detector integrated scattering intensities of a latex particle in water with diameter $d=1.36 \mu$ m; penetration depth $\zeta=300 \mathrm{~nm}$ $\left(\theta=62.5396^{\circ}\right)$.

To summarize these findings, we would like to mention that two different methods have been applied to model existing conditions of TIRM experiment. Simulation results of NFM-DS and DSM demonstrate perfect agreement between each other and also with measurements. In future we plan to go further and apply our methods to model different kinds of particles on substrate used for TIRM and investigate the dependence of the superimposed oscillation (phase, amplitude) on experimental parameters like particle size, incident angle and polarization.

\section{Conclusion}

In this paper the null-field method and discrete sources method have been applied to model evanescent wave scattering by a particle for TIRM experiments. The numerical algorithms of DSM and NFM-DS have been modified to take into account features of real measurement setup. Objective response and scattering crosssection have been calculated. Numerical results of both methods demonstrated good agreement with each other and with experimental data.

\section{Acknowledgments}

We gratefully acknowledge funding of this research by Deutsche Forschungsgemeinschaft (DFG) and the Russian Foundation for Basic Research (RFBR).

\section{References}

[1] Prieve DC, Luo F, Lanni F. Brownian motion of a hydrosol particle in a colloidal force field. Faraday Disc Chem Soc 1987;83:297 307.

[2] Helden L, Koenderink GH, Leiderer P, Bechinger C. Depletion potentials induced by charged colloidal rods. Langmuir 2004;20:5662 5.

[3] Walz JY. Measuring particle interactions with total internal reflection microscopy. Curr Opin Colloid Interface Sci 1997;2:600 6.

[4] Prieve DC. Measurement of colloidal forces with TIRM. Adv Colloid Interface Sci 1999;82:93 125.

[5] Bike SG. Measuring colloidal forces using evanescent wave scattering. Curr Opin Colloid Interface Sci 2000;5:144 50.

[6] Eremin Y, Orlov N, Sveshnikov A. In: Wriedt T, editor. Generalized multipole techniques for electromagnetic and light scattering. Amsterdam: Elsevier; 2002. p. 39.

[7] Eremin Y. The method of discrete sources in electromagnetic scattering by axially symmetric structures. J Commun Technol Electron 2000;45:269 80 
[8] Doicu A, Eremin Y, Wriedt T. Light scattering by systems of particles. Null field method with discrete sources theory and programs. Berlin: Springer; 2006.

[9] Eremin Y, Wriedt T. Large dielectric particle in an evanescent wave field near a plane surface. Opt Commun 2000;214:39.

[10] Doicu A, Eremin Y, Wriedt T. Convergence of the t matrix method for light scattering from a particle on or near a surface. Opt Commun 1999;159:266 77.

[11] Doicu A, Eremin Y, Wriedt T. Scattering of evanescent waves by a sensor tip near plane surface. Opt Commun 2001;190:5 12.

[12] Helden L, Eremina E, Riefler N, Hertlein C, Bechinger C, Eremin Y, et al. Single particle evanescent light scattering simulations for total internal reflection microscopy. Appl Opt 2006;45:7299 308.

[13] Wiscombe W. Improved Mie scattering algorithm. Appl Opt 1990;19:1505 9. 